\title{
Coherent population trapping: its physics and historical roots
}

\author{
A.M. Shalagin* \\ Institute of Automation and Electrometry SB RAS, 630090 Novosibirsk, Russia
}

\begin{abstract}
There presented basic information on coherent population trapping (CPT) and related phenomenon of electromagnetically induced transparence, 'dark' and 'bright' (anomalous absorption) states is presented. Spectrum transformation is considered in probe field spectroscopy for $\Lambda$-scheme in various settings. The close relation is revealed between the effect of CPT in the manifold of magnetic sublevels and the phenomenon of optical pumping (optical orientation and optical alignment). CPT is shown to be generalized to the case of several transitions with a common upper level and with the corresponding number of light fields each being in quasiresonance with its 'own' transition.
\end{abstract}

When discussing the phenomenon known as coherent population trapping (CPT) or electromagnetically induced transparency (EIT), they usually cite [1] as the first experimental observation and [2] as the first review of theory and experiments from 1976 up to that date. CPT is still in focus due to its expected applications in fine optical frequency standards, ultra-sensitive magnetometry, high-resolution spectroscopy etc.

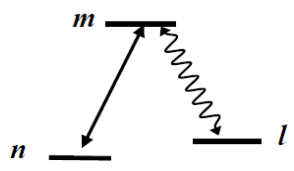

Fig.1

The essence of CPT is as follows. Let us consider three-level $\Lambda$ scheme (see Fig.1) where the lower levels $l$ and $n$ do not relax and both the transitions $m-n, m-l$ are driven by two quasi-resonant monochromatic fields. In the most simple case, one of the fields (pump field) has arbitrary intensity and the second is weak (probe field). The absorption spectrum of the probe field

$$
I(\varepsilon)=I_{0} \frac{\Gamma_{m l}^{2}}{\Gamma_{m l}^{2}+\left(\Omega+\varepsilon-|G|^{2} / \varepsilon\right)^{2}} ; \quad \varepsilon \equiv \Omega_{\mu}-\Omega .
$$

Here $\Omega$ and $\Omega_{\mu}$ - the frequency detunings of the pump and probe fields with regard to the respective transitions, $\Gamma_{m l}-$ the $m-l$ polarization decay rate. It is evident that under the condition of equal detunings $(\varepsilon=0)$ the probe field's absorption is absent. That one of the pump field is absent as well due to the total population being at $l$. This is the CPT phenomenon: the disappearance of absorption of fields which drive the adjacent transitions. Examples of absorption spectrum $I(\varepsilon)$ are depicted in Fig. 2. CPT yields narrow resonances. When $\Omega=0$ (Fig. 2a) the resonance appears as a narrow gap in center of a broad line (with

\footnotetext{
*Corresponding author: shalagin@iae.nsk.su
} 
FWHM $\Gamma_{m l}$ ), for large $\Omega$ (Fig. $2 c$ ) it is still a more narrow peak slightly shifted from $\Omega_{m}=$ $\Omega$. This peak can be used in high-resolution spectroscopy.
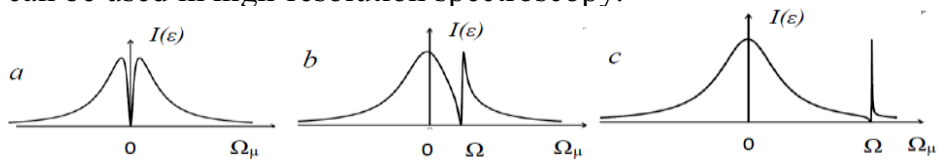

Fig. 2

The condition $\Omega_{\mu}=\Omega$ provides zero absorptions for arbitrary relation between the fields' intensities. The level $m$ is then empty whereas the populations and their coherence

$$
\rho_{n n}=\frac{\left|G_{\mu}\right|^{2}}{|G|^{2}+\left|G_{\mu}\right|^{2}} N ; \quad \rho_{l l}=\frac{|G|^{2}}{|G|^{2}+\left|G_{\mu}\right|^{2}} N ; \quad \rho_{n l}=\frac{-G^{*} G_{\mu}}{|G|^{2}+\left|G_{\mu}\right|^{2}} N ; \quad\left|\rho_{n l}\right|=\sqrt{\rho_{n n} \rho_{l l}} .
$$

The populations depend on the fields' intensities (more precisely, on the Rabi frequencies). The inter-level coherence is maximal. This gives rise to the name of CPT.
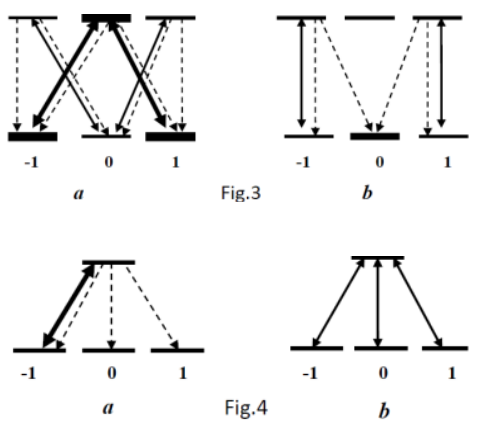

Now we consider the case of polarized radiation and levels with magnetic degeneracy. The transitions between triply degenerate levels (with unit angular momentum) due to linearly polarized radiation are shown in Fig. 3. When the quantization axis is directed along the radiation wave-vector the transitions are those in Fig. 3a. The solid arrows show induced transitions, the dotted arrows stand for spontaneous transitions. Note that transitions between sublevels with zero projection of angular momentum are strictly forbidden. The induced and spontaneous transitions completely depopulate the central sublevel of the ground state as well as the side sublevels of the excited state. As a result, only 'super-solid' transitions and sublevels will contribute to interaction. That means that the standard $\Lambda$-scheme of CPT is extracted. The fields driving the adjacent transitions have equal detunings. Hence, there must be zero absorption. This assertion gets almost straightforward if the quantization axis is directed along the electric field. In this case the scheme of transitions is depicted in Fig. $3 b$. The induced and spontaneous transitions cause the optical pumping of the central ground sublevel, so that the absorptions disappears. This example shows CPT and optical pumping being the same in their origin. On the other hand, optical pumping had been already known before the laser invention. It brought Nobel Prize to A. Kastler. We ought to give CPT researchers credit for they pointed a proper choice of basis clarifies the phenomenon as the formation of a specific superposition state which decuples the system from interaction with the fields (the so-called 'dark' state).

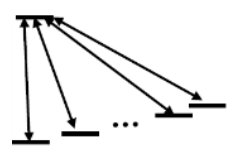

Fig. 5

Let us examine another interesting situation with degenerate levels when transitions from triply degenerate ground level to non-degenerate excited one is induced by circularly polarized radiation (see Fig. 4). With the quantization axis along the wave-vector the optical transitions will eventually depopulate the left ground sublevel (see Fig. $4 a$ ). The central and right sublevels get populated (optical orientation [3]), and the field's absorption disappears. The same phenomenon, being considered with an orthogonal quantization axis, looks like an interaction of the system with three fields with the same frequency (see Fig. 4b). A more general situation is imaginable with any number of transitions from a common upper level and with the corresponding number of driving fields (Fig. 5). If at least two fields get equal detunings, the medium will be transparent for all the fields' manifold.

\section{References}

1. G. Alzetta et al., Nuovo Cimento Soc. Ital. Fis. B 36, 5 (1976)

2. E. Arimondo, Prog. Opt. 35, 257 (1996)

3. A. Kastler, Preprint les Prix Nobel en 1966 (Nobel Foundation, Stockholm, 1967) 